\title{
Research Paper \\ Relationship Between Coping Strategies and Locus of Control With the Anxiety of Death in Old People
}

${ }^{*}$ Hadi Hashemi Razini ${ }^{1}$, Shahab Baheshmat Juybari ${ }^{1}$, Maryam Ramshini ${ }^{2}$

1. Department of General Psychology, Faculty of Psychology and Education, Kharazmi University, Tehran, Iran.

2. Department of Psychology and Education of Exceptional Children, Faculty of Humanities and Social Sciences, Science and Research Branch, Islamic Azad University, Tehran, Iran.

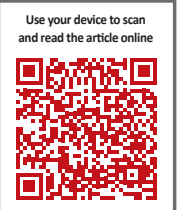

Citration: Hashemi Razini H, Baheshmat Juybari Sh, Ramshini M. [Relationship Between Coping Strategies and Locus of Control With the Anxiety of Death in Old People (Persian)]. Iranian Journal of Ageing. 2017; 12(2):232-241. http://dx.doi. org $/ 10.21859 /$ sija- 1202232

\section{http://dx.doi.org/10.21859/sija-1202232}

Received: 09 Feb. 2017

Accepted: 26 Apr. 2017

Key words:

Death anxiety, Cop-

ing strategies, Locus

of control, Older

adults

\section{A B STRACT}

Objectives The prevalence of death anxiety in older adults is high. Thus, the aim of the present study was to explore the relationship between coping strategies and locus of control with death anxiety in older adults.

Methods \& Materials In this descriptive and cross-sectional study, 113 elderly adults older than 60 years were recruited from the city of Tehran via available sampling method. For data collection, the Coping Strategies Inventory, Locus of Control Inventory and Death Anxiety Scale were employed. Then, the data were analyzed using SPSS 21 software and statistical tests such as Pearson correlation coefficient and Stepwise regression.

Results The mean (SD) age of the participants was 65.19(3.93) years. The mean scores of problem-focused coping, emotion-focused coping, avoidance-focused coping, internal locus of control, external locus of control, and death anxiety were 48.20 $\pm 7.45,50.93 \pm 6.92,50.39 \pm 8.67,17.85 \pm 5.70,61.51 \pm 6.08$ and $8.67 \pm 2.31$, respectively. Based on study findings, there was a statistically significant relationship between coping strategies $(P<0.05)$ and locus of control $(P<0.05)$ with death anxiety among older adults. The results of stepwise regression analysis indicated that avoidance and emotion oriented coping and external locus of control significantly predict death anxiety $(P<0.01)$.

Conclusion Death anxiety is one of the key factors in the mental health of older adults. With regard to our findings, coping strategies and locus of control have an important role in death anxiety in older adults. Therefore, by designing psychological interventions based on coping strategies and changing locus of control from external to internal, death anxiety among the older adults can be improved.

\section{Extended Abstract}

\section{Objectives}

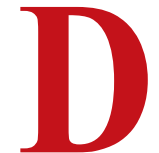

eath is a source of fear and anxiety for the older people; this is because death threatens the meaning of existence and ends life.
Due to the deterioration and loss associated with aging, it is expected that death anxiety increases as age increases A review of the literature shows that there are several factors contributing to the anxiety of death such as locus of control, and accordingly, coping strategies have been investigated in several studies. Given the uncertainty, uncontrollability and irreversible process of death, anxiety

\section{* Corresponding Author:}

Hadi Hashemi Razini, PhD

Address: Department of General Psychology, Faculty of Psychology and Education, Kharazmi University, Tehran, Iran.

Tel: +98 (21) 44463621

E-mail: hadihashemirazini@yahoo.com 
is mainly associated with avoidance and emotion-focused strategies. Problem-oriented strategy known as trying to change the environment, does not seem to be associated with death anxiety. Furthermore, studies show that internalization is associated with more positive results, compared to externalization. For example, a sense of personal control is negatively accompanied by high perceived stress, negative mood and death anxiety. Therefore, the current study aimed to investigate the predictive role of coping strategies and locus of control in old people's death anxiety.

\section{Methods \& Materials}

The current study is cross-sectional correlational study. The study sample comprised of all aged people in Tehran selected from public places such as neighborhood house, parks and cultural centers. To estimate the sample size, the proposed formula of Tabachnick and Fidel (2007) was used. Accordingly, the sample size was determined to be 106 using this formula. To ensure that the questionnaires are returned and also the possibility of partial completion of the questionnaire, and to prevent sample loss, the sample size was increased by $10 \%$ and finally 120 questionnaires were distributed to the population. To select the subjects needed for research, criteria such as the age of 60 years and above, lack of acute and chronic debilitating physical and mental diseases, lack of cognitive disorder and the ability to read and write were considered for inclusion. Due to the specific characteristics of the study population and inclusion criteria, convenient sampling was used for sample selection. Eventually, after collecting the questionnaires and removing the faulty questionnaires, 113 questionnaires were analyzed. In order to observe moral considerations, informed consent to participate in the study was obtained from the participants. This study had no mental and physical harm on the participants, and all the participants were ensured that their information would remain strictly confidential. To collect the data, the Coping Strategies Scale, Multidimensional Locus of Control Scale, and Death Anxiety Scale (DAS) were distributed among sample subjects. It should be noted that research instruments were completed under close observation of the co-author at the place of the study. Data analysis was done using SPSS software version 21, and descriptive statistical methods were used to calculate the central tendency and variability indexes. Also, to evaluate the hypotheses, the Pearson product-moment correlation coefficient and stepwise regression analysis were used.

\section{Results}

The average age of participants was $65.19 \pm 3.93$ years. The mean (standard deviation) of problem-oriented coping, emotion-focused coping, avoidance coping, locus of control, external locus of control, and death anxiety were $48.20 \pm 7.45,50.93 \pm 6.92,50.39 \pm 8.67,17.85 \pm 5.70$, $61.51 \pm 6.08$, and $8.67 \pm 2.31$, respectively. In order to investigate the role of coping strategies and locus of control in explanation and prediction of death anxiety in the old

Table 1. Results of stepwise regression analysis in prediction of death anxiety based on the predictor variables

\begin{tabular}{|c|c|c|c|c|c|c|c|c|c|}
\hline \multirow{2}{*}{ Step } & \multirow{2}{*}{ Predictive Variables } & \multirow{2}{*}{ B } & \multirow{2}{*}{ ß } & \multirow{2}{*}{$\mathbf{R}$} & \multirow{2}{*}{$\mathbf{R}^{2}$} & \multirow{2}{*}{$\mathbf{F}$} & \multirow{2}{*}{ Sig. } & \multicolumn{2}{|c|}{ Linearity Assumption } \\
\hline & & & & & & & & Tolerance & Variance Inflation \\
\hline \multirow{3}{*}{1} & Fixed & 2.089 & - & \multirow{3}{*}{0.281} & \multirow{3}{*}{0.079} & \multirow{3}{*}{9.53} & \multirow{3}{*}{0.003} & \multirow{3}{*}{-} & \multirow{3}{*}{-} \\
\hline & & & & & & & & & \\
\hline & External control & 0.107 & 0.281 & & & & & & \\
\hline \multirow{3}{*}{2} & Fixed & 6.047 & - & \multirow{3}{*}{0.364} & \multirow{3}{*}{0.132} & \multirow{3}{*}{8.39} & \multirow{3}{*}{0.000} & - & - \\
\hline & External control & 0.094 & 0.264 & & & & & 1.023 & 0.978 \\
\hline & Avoidance coping & -0.062 & -0.234 & & & & & 1.023 & 0.978 \\
\hline \multirow{4}{*}{3} & Fixed & 9.895 & - & \multirow{4}{*}{0.404} & \multirow{4}{*}{0.163} & \multirow{4}{*}{7.09} & \multirow{4}{*}{0.000} & - & - \\
\hline & External control & 0.080 & 0.209 & & & & & 0.936 & 1.068 \\
\hline & Avoidance coping & -0.060 & -0.227 & & & & & 0.976 & 1.024 \\
\hline & Emotion coping & -0.060 & -0.180 & & & & & 0.953 & 1.049 \\
\hline
\end{tabular}


people, the Pearson product-moment correlation coefficient and stepwise regression analysis were used. These tests have assumptions as parametric tests, which were measured and evaluated before inferential analysis.

The results of the assumption tests showed that these tests can be used to evaluate the hypothesis of the research. Pearson correlation coefficients showed that the avoidance and emotion-focused strategies have a significant negative correlation with death anxiety. In contrast, problemfocused coping strategies showed a significant negative correlation with death anxiety. It was also found that the external locus of control has a significant negative relationship with death anxiety, and the locus of control has a positive relationship with death anxiety. Stepwise regression analysis (Table 1) shows that in the first step, the first variable that was entered into the analysis and remained was the external control; this means that it was the most effective variable compared to other predictive variables.

In the second step, after external control variable, avoidance coping variable entered into the equation. In the second step, according to the coefficient of determination obtained, it can be stated that about $13 \%$ of the death anxiety of old people was due to the two variables of external control and avoidance coping strategies. In the third step, the last variable that entered into the analysis was predictor variable of emotion-focused coping. The correlation coefficient calculated in this step was 0.404 , and the coefficient of determination was 0.163 . The F obtained shows that the calculated regression model is significant at a confidence level of $99 \%$. According to the coefficient of determination, it seems that 0.16 of variations of the variable can explain the criterion for death anxiety in old people by three predictive variables of external control, avoidance coping, and emotion-focused coping. The amount of beta shows that external control can directly predict death anxiety and avoidance coping and emotion-focused predict it in the opposite direction. In other words, with the increasing avoidance and emotion-focused coping, the level of death anxiety in old participants was reduced, and the more the external control increases, the more the death anxiety also increases along with it.

\section{Conclusion}

Although in the research literature, especially studies by Folkman et al., there is a consensus on the negative impact of avoidance and emotion-focused coping, there are consequences and emotional events and coping strategies known as adaptive strategies to deal with stressful events. However, these assumptions are true when stress- ful events are manageable. In explaining this conclusion, it seems that old people who use avoidance and emotionfocused strategies deal with the ambiguous nature of the coming death more adoptively. Furthermore, Levenson (1973) believed that people with an internal locus of control have control over the consequences of an event go out of the cycle of repetitive thoughts easier. When people believe that they can cope with a stressful event, their concern is decreased and anxiety levels are also reduced on using effective coping strategies. As a result, it can be said that the more the external locus of control in old people, the less their ability to control racing repetitive thoughts to death.

\section{Acknowledgments}

This research did not receive any specific grant from funding agencies in the public, commercial, or not-forprofit sectors.

\section{Conflict of Interest}

All authors certify that this manuscript has neither been published in whole nor in part nor being considered for publication elsewhere. The authors have no conflicts of interest to declare. 


\title{
بررسى ارتباط راهبردهاى مقابله و منبع كنترل با اضطراب مرتى در سالمندان
}

\author{
"هادى هاشمى رزينى'، شهاب باحشمت جويبارى'، مريم رامشينى' \\ 1- كروه روانشناسى عمومى، دانشكده روانشناسى و علوم تربيتى، دانشكاه خوارزمى، تهرانه ايران.

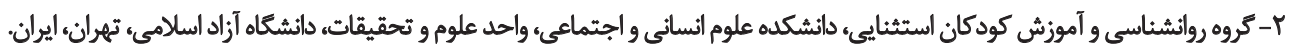

\begin{abstract}
حكيد
الهدافق اضطراب مرك در افراد سالمند شايع است. هدف از يرؤشش حاضر بررسى ارتباط راهبردهاي مقابله و منيع كنترل با اضطراب

مركى در سالمثلدان است.

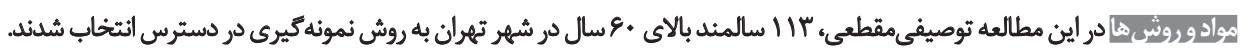

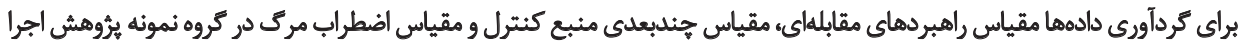

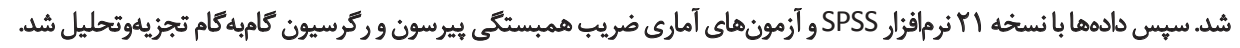

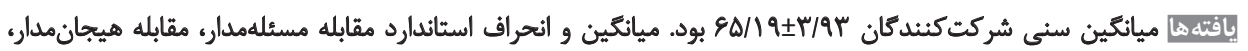

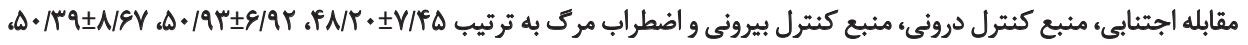

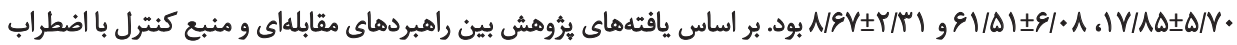

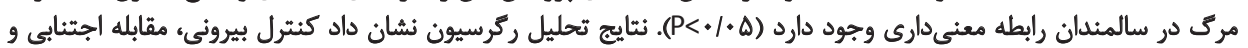

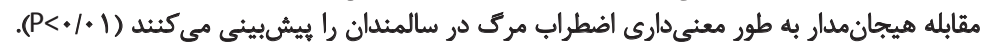

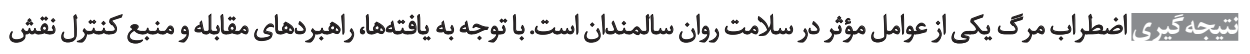

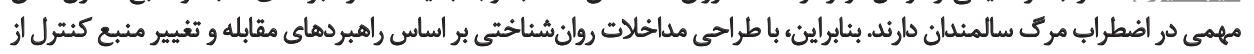

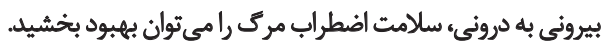

تاريخ دريافت: ال بهمن هوبا

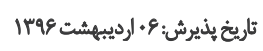

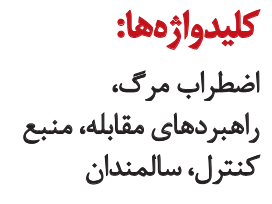

كليدواثرهها:

اضطراب مركى،

كنترل، سايمندان مقابلن

كستردهاي در دوره سالمندى بررسى شده است [ه]].

مقدمه - - مق

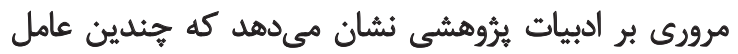

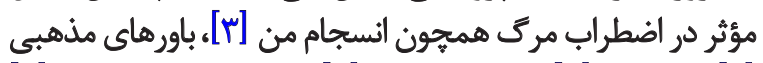

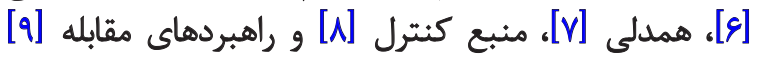

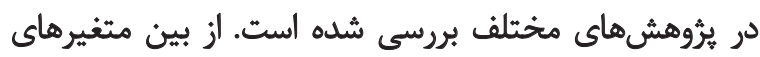

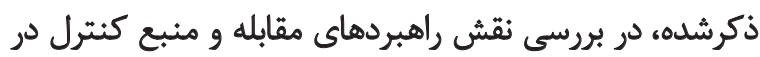

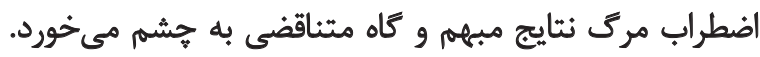

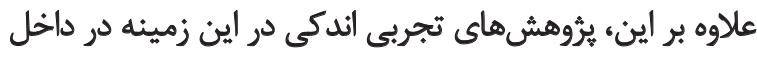

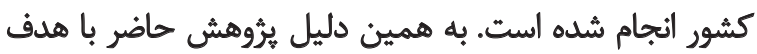

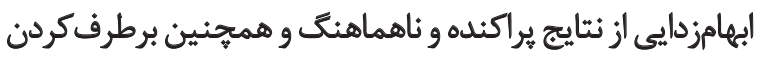
فقر اطلاعاتى در اين زمينه انجام شده است.

در سالهاى احير روانشناسى مثبت كرا با اقبال كسترده

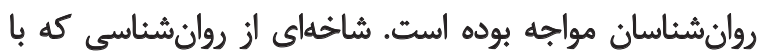

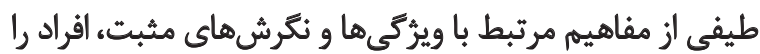

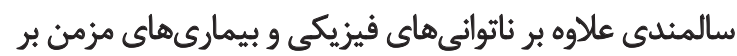

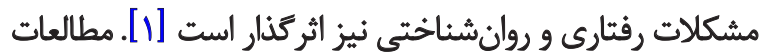

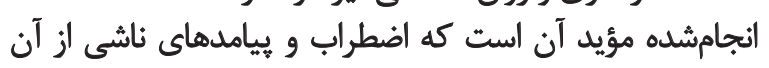

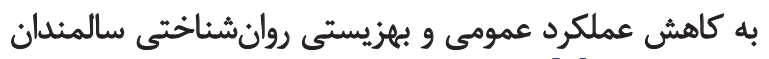

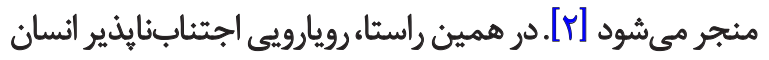

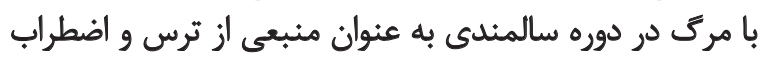

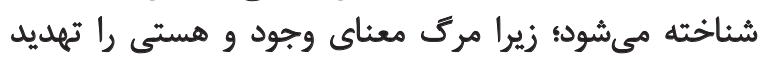

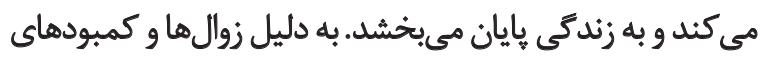

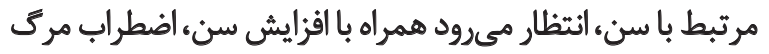

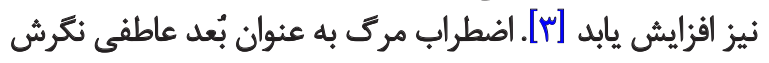

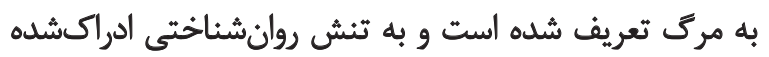

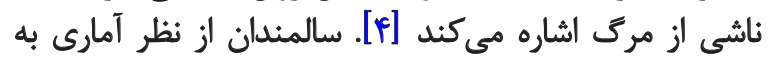

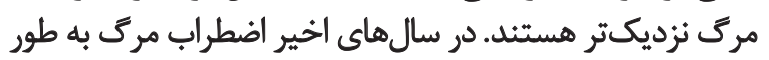


سامرين و زوبير" [19] در بررسى كاركنان يليس نشان دادند

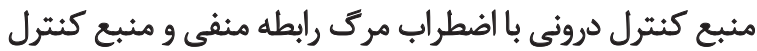

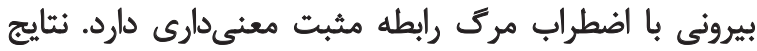

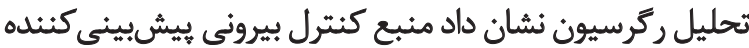

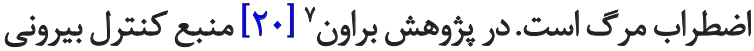

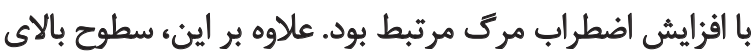

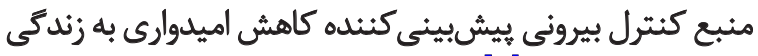

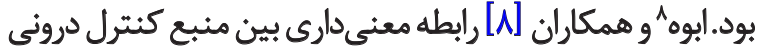

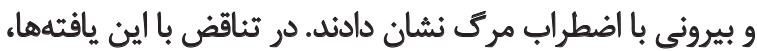

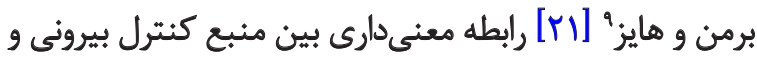
اضطراب مرك به دست نياوردند.

ادبيات بروهشى نشان مى دهد نقش راهبردهاى مقابله و منبع

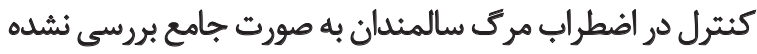
است و يثوهش هاى صور

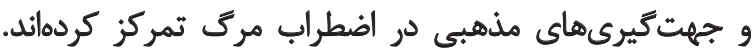

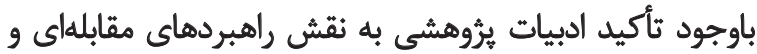

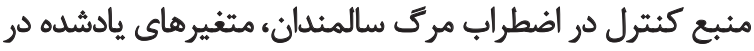

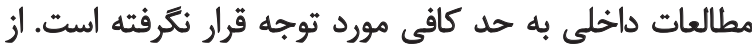

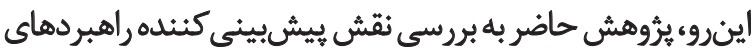

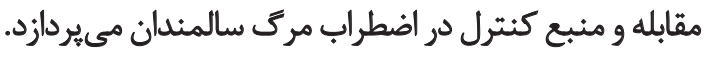

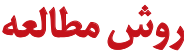

روش ثيروهش حاضر، توصيفىمقطعى از نوع همبستخى است.

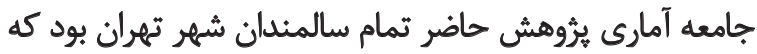

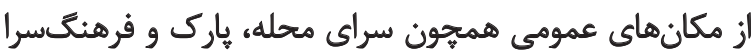

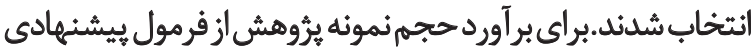

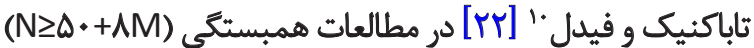

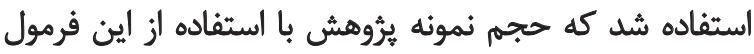

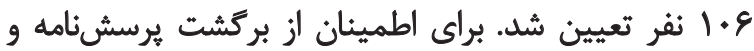

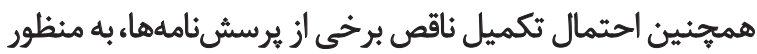

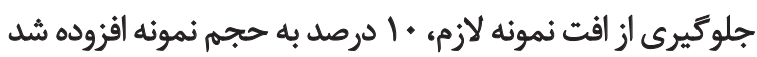

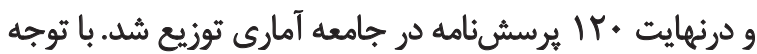

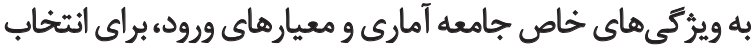

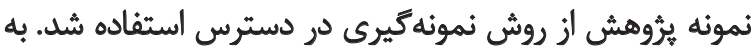

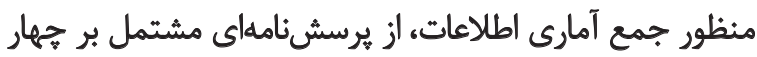

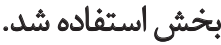

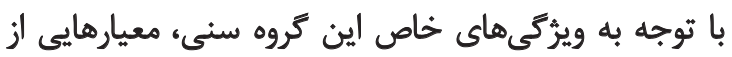
قبيل سن •و سال به بالا، نداشتن بيمارىهائ كروه حادي، معياروايى مزمن

6. Samreen and Zubair

7. Brown

8. Aboh

9. Berman and Hays

10. Tabakhnick and Fidell
قادر مي سازد با روشهاى سالمتر و سازندهترى با موقعيتهاى

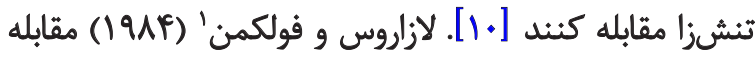

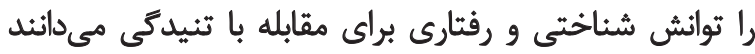

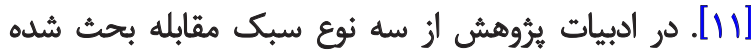

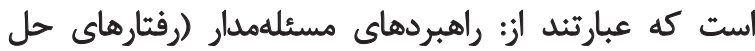

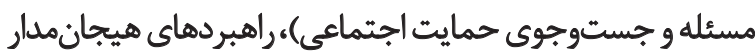

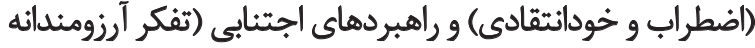

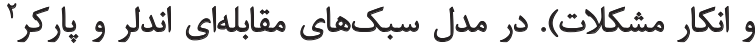

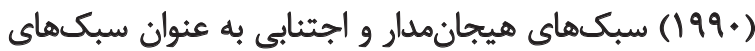

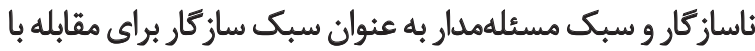

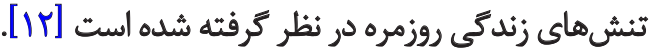
با توجه به ابهام، كنترلنايذيرى و غيرقابل تغيير بودن فرايند

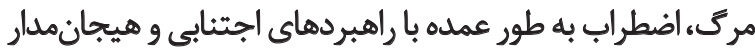

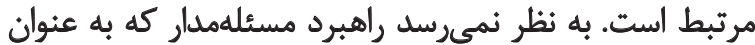

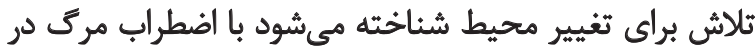

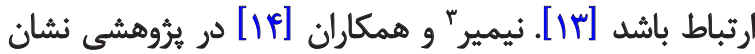

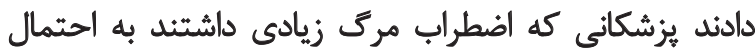

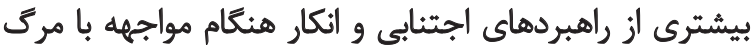

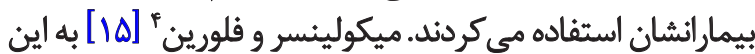

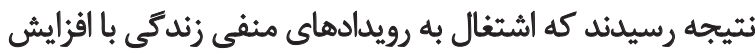

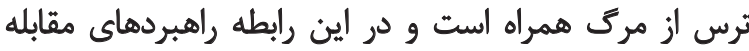

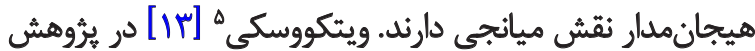

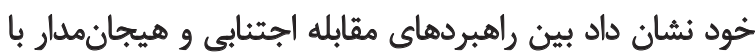
نغرش به مرى رابطه معنى دارى وجود دارد. از سوى ديكر، منبع كنترل به تفاوت باورهايي اشاره دارد كه

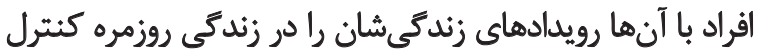

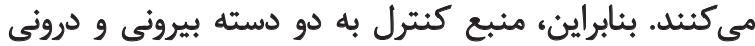

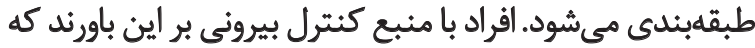

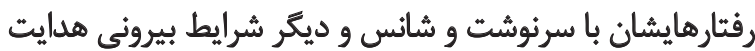

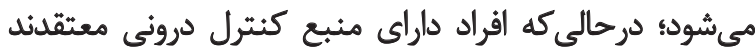

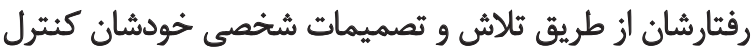

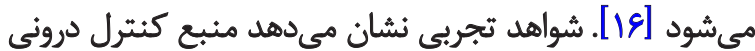

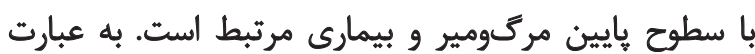

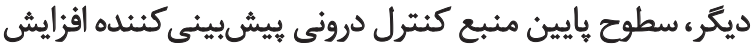

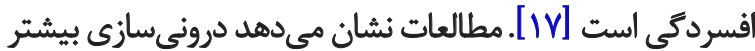

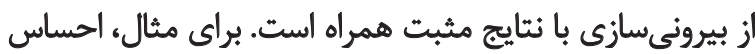

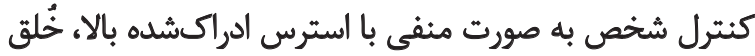

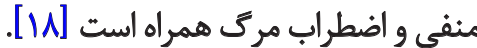

1. Lazarus and Folkman

2. Endler and Parker

3. Neimeyer

4. Mikulincer and Florian

5. Wittkowski 


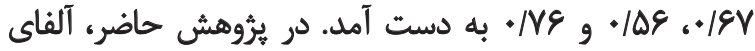

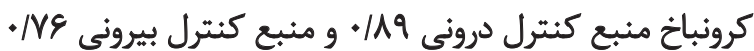

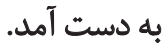

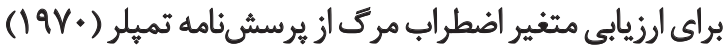

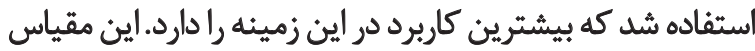

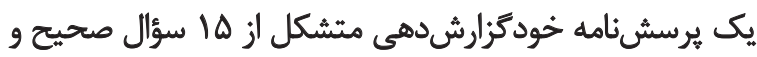

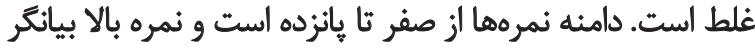

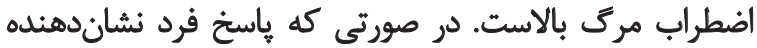

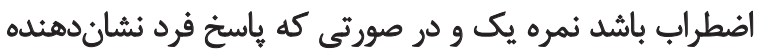

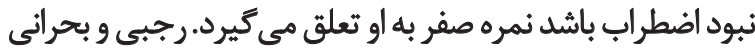

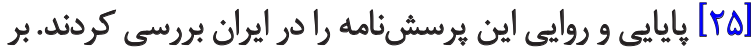

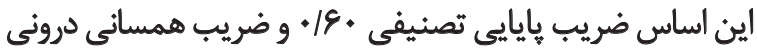

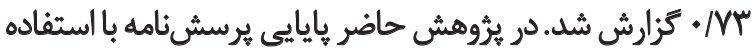

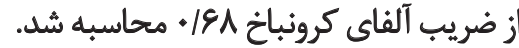

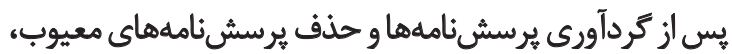

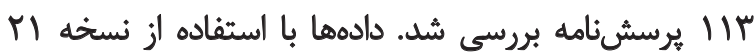

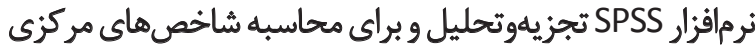

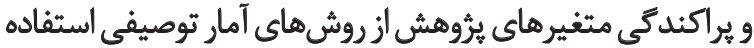

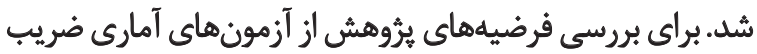

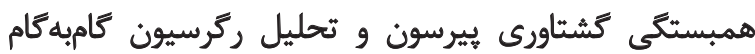

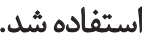

\section{يافتهها}

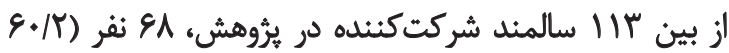
9.

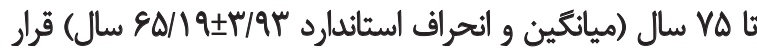

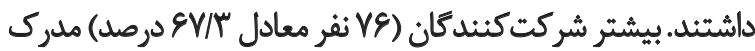

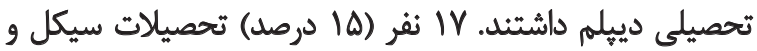

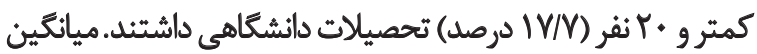

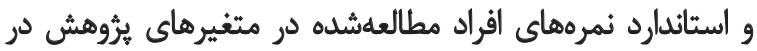

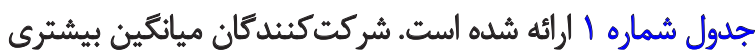

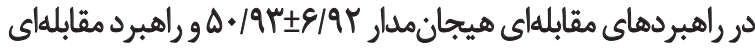

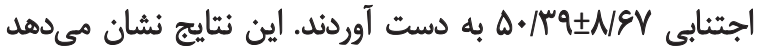

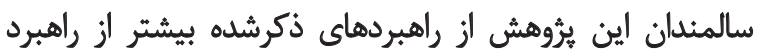

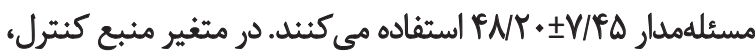

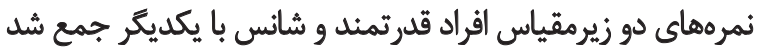

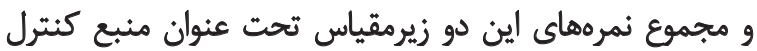

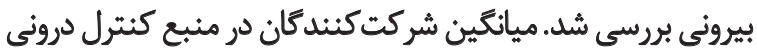

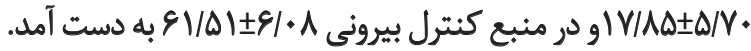

به منظور بررسى نقش راهبردهاي مقابلهاى و منبع كنترل

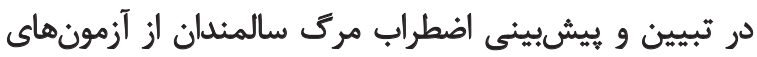

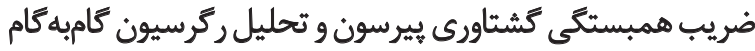

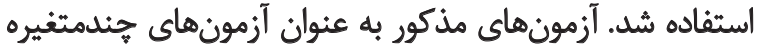

ناتوان كنيده جسمى و روانى، نداشتن اختلال شناختى و توانايى

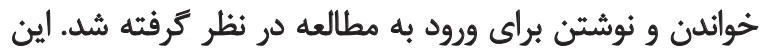

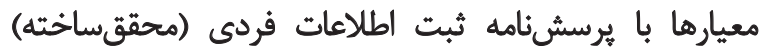

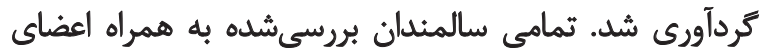

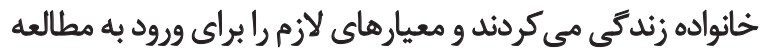

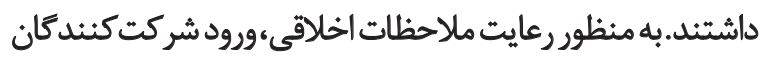

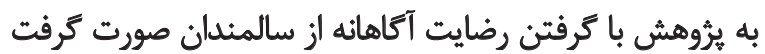

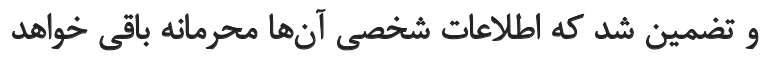

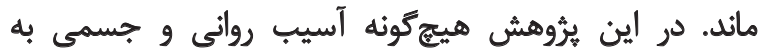

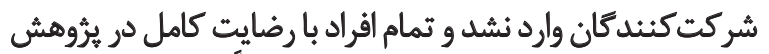

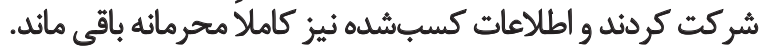

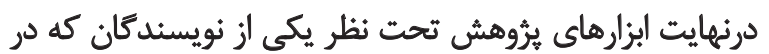

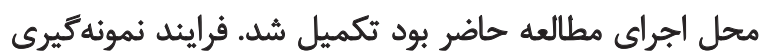

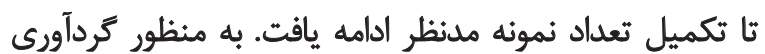

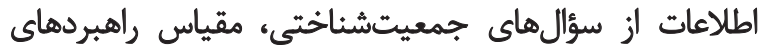

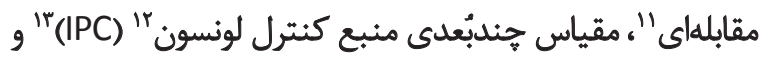

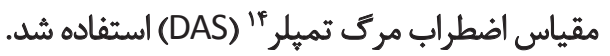

مقياس راهبردهاي مقابلهاي را اندلر و ياركر (•99 (199) ساختهاند

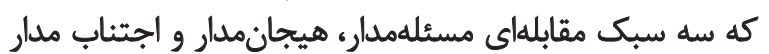

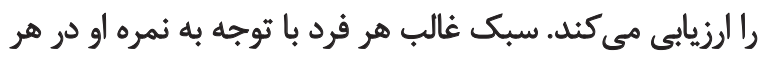

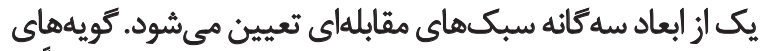

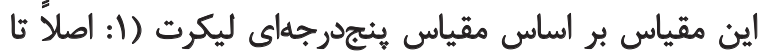

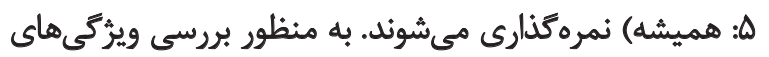

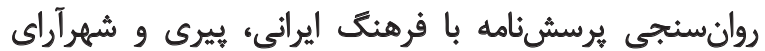

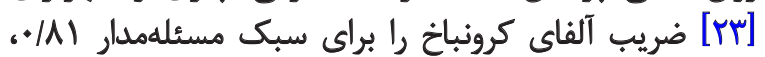

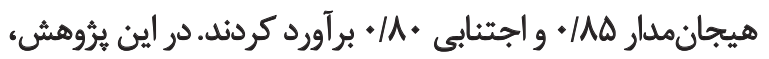

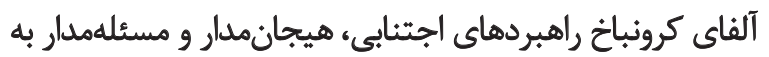

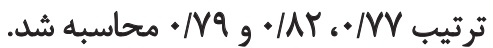

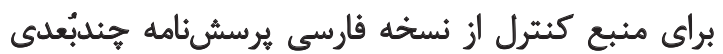

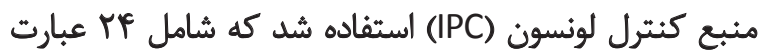

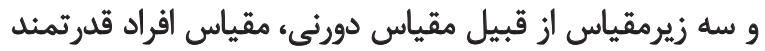

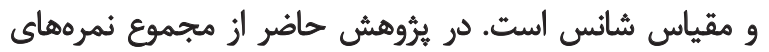

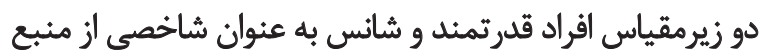

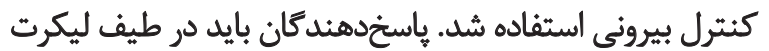

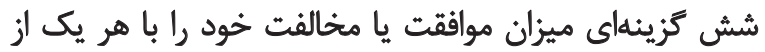

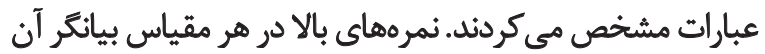

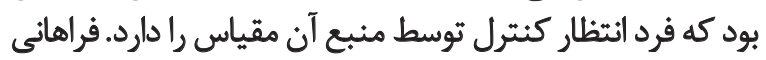

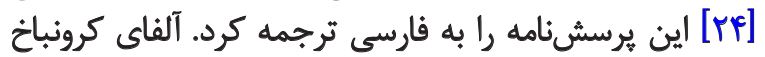
براى زيرمقياسهاى درونى، افراد قدرتمند و شائس به ترتئ كرديب

11. Coping Inventory for Stressful Situations (CISS) 12. Levenson Multidimensional Locus of Control Scales 13. Internality, Powerful Others, and Chance Scales (IPC) 14. Templer Death Anxiety Scale (DAS) 
جدول ا. شاخصهاي مركزى و يراكندكى متغيرهاي بئوهش

\begin{tabular}{|c|c|c|c|c|c|}
\hline انحرافمعيار & مياتكين & بيشينه & كمينه & تعداد & هتغير \\
\hline$V / P \Delta$ & PNT. & go & ra & $11 \%$ & مقابله مسثلهمدار \\
\hline g/ar & $0 . / 9 Y$ & eq & r. & $11 \%$ & مقابله هيجانمدار \\
\hline NeV & $0 . / 1 \% q$ & eV & MA & $11 \%$ & مقابله اجتنتابي \\
\hline$\Delta / V$ & IV/NA & Tr & 9 & $11 \%$ & كتترل درونى \\
\hline $91 \cdot 1$ & $81 / 01$ & ve & ra & $11 \%$ & كنترل بيرونى \\
\hline$T / \mu$ & NEV & ir & f & $11 \%$ & اضطراب مرك \\
\hline
\end{tabular}

郔

جدول ب. ماتريس همبستكي متغير هاى يُروهش

\begin{tabular}{|c|c|c|c|c|c|c|c|}
\hline 8 & $\Delta$ & $f$ & $r$ & $r$ & 1 & مثغير & \\
\hline & & & & & 1 & مقابله مسثلهمدار & 1 \\
\hline & & & & 1 & $-\cdot / 0 \cdot p=$ & مقابله هيجان ملار & r \\
\hline & & & 1 & $\cdot 1 \cdot v$ &.$- / 118$ & مقابله اجتنابي & r \\
\hline & & 1 &.$|+\Delta|$ & $\cdot / r+. *$ & $-\bullet / \pi T \Lambda^{* *}$ & كتتل دروئى & $p$ \\
\hline & 1 & $-* / M F$ &.$- / 10$ & $-\cdot / M I M$ &.$/ M V^{*}$ & كنترل بيروني & $\Delta$ \\
\hline 1 & . $(\text { YA })^{* *}$ &.$- / 194$ & $-+/ 4 \gamma+\cdots$ & $-* / \pi r_{*}$ &.$/ 191^{*}$ & اضطراب مرى & $\varepsilon$ \\
\hline
\end{tabular}

ماتريس ضريب همبستكَي بيرسون (جدول شماره Y) نشان داد

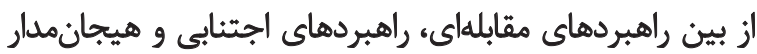

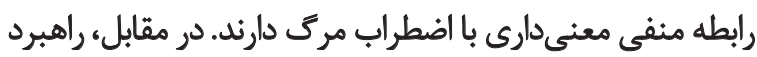

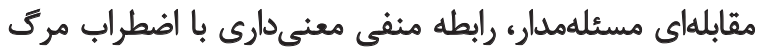
نشان داد. بررسى ضرايب همبستئى بهدست آمده نشان مي مدهدي

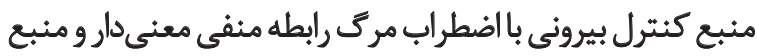

كنترل درونى با اضطراب مرى رابطه مثبت معنى دارى دارد

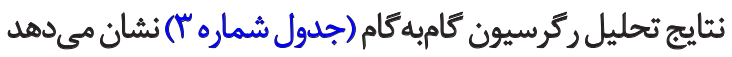
در كام اول اولين متغيرى كه وارد تحليل شد شو و باقيى مائد، كثترل

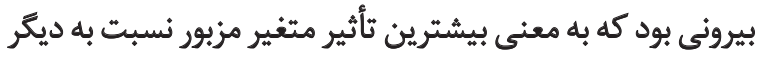

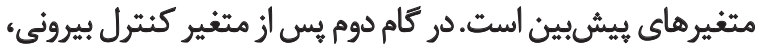

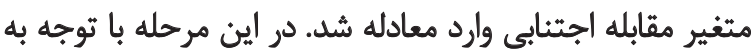

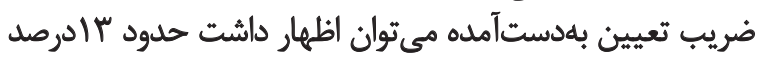

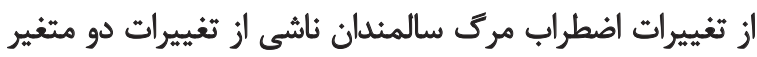

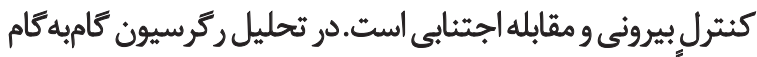

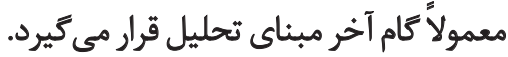

در كام سوم آخرين متغيرى كه وارد ثحليل شد متغير

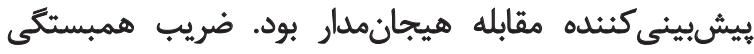

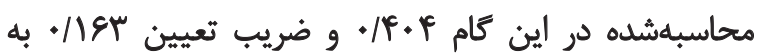

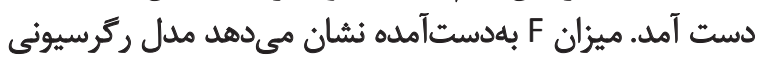

يّيش فرض هايي داشتند كه بهيش از تحليل استنباطي سنجش و

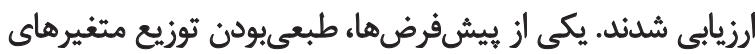

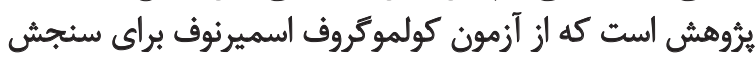

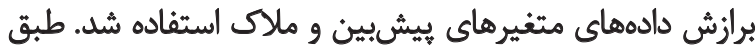

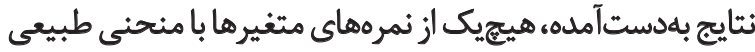

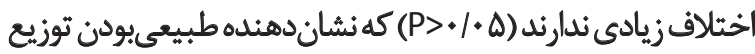

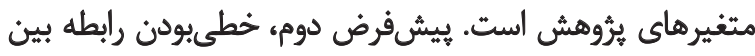

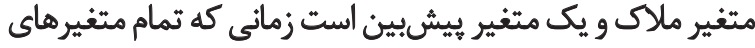

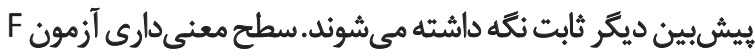

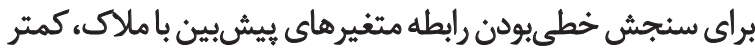

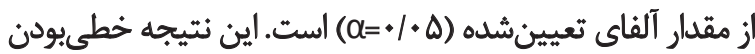

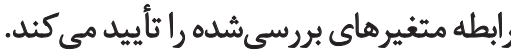

ميشفرض ديكر تحليل ركرسيون عدم همبستكى زياد بين

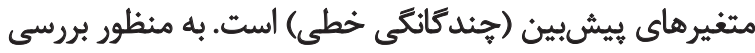

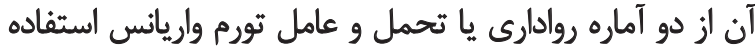

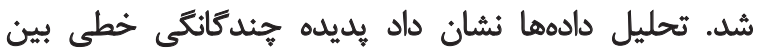

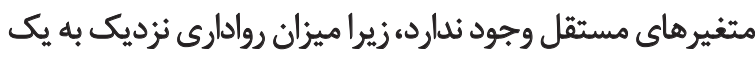

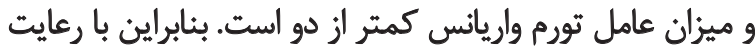

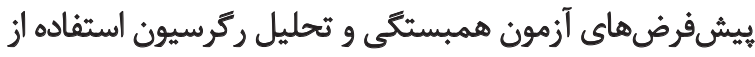

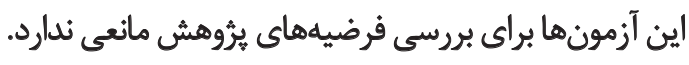


جدول ऍ. نتايج تحليل ركرسيون كَامبه كام در بيشبينى اضطراب مرك بر اساس متغيرهاى يُيشبين

\begin{tabular}{|c|c|c|c|c|c|c|c|c|c|}
\hline \multicolumn{2}{|c|}{ مفروضه هيرخطى } & \multirow{2}{*}{ Sig. } & \multirow{2}{*}{$\mathbf{F}$} & \multirow{2}{*}{$\mathbf{R}^{r}$} & \multirow{2}{*}{$\mathbf{R}$} & \multirow{2}{*}{$\beta$} & \multirow{2}{*}{ B } & \multirow{2}{*}{ مثغير هاى ييشبين } & \multirow{2}{*}{ (5 } \\
\hline تورم واريانس & تحمل & & & & & & & & \\
\hline $\begin{array}{c}- \\
1 / \ldots\end{array}$ & $\begin{array}{c}- \\
11 . .\end{array}$ & 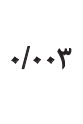 & Q/Dr & $.1 . \mathrm{V9}$ & $. / T A \mid$ & $\begin{array}{c}- \\
.|M A|\end{array}$ & $\begin{array}{l}r / 19 \\
.11 \cdot V\end{array}$ & كثترل بيرونيت & 1 \\
\hline - & - & & & & & - & eleP & ثابت & \\
\hline.$/ 9 V A$ & $V \cdot \pi$ & $\%$ & NFq & (IIT & . Iret & - KEP & .1 .94 & كتترل يبيرونى & $r$ \\
\hline.$/ 9 V A$ & $V \cdot m$ & & & & &.$- / M T P$ & -.1 .84 & مقابله اجتنابي & \\
\hline- & - & & & & & - & V/194 & ثابت & \\
\hline $1 / .81$ & & 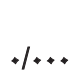 & $V / .9$ & . MET & $\cdot p \cdot+f$ & $+/ r+9$ & $1+1$ & كتترل بيرونى & $r$ \\
\hline $1 / \cdot \pi f$ &.$/ 9 v 8$ & & & & &.$- / M T V$ & -+1.8 & هقابله اجتنابى & \\
\hline $1 /+p q$ &.$/ 9 \Delta r$ & & & & &.$- / 11$ & $-* 1+8$ & مقابله هيجاني & \\
\hline
\end{tabular}

ك

با افزايش ترس از مرك همراه است اما مقابله اجتئابى ثا حدودى

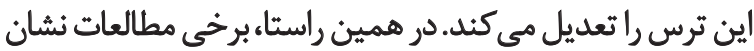

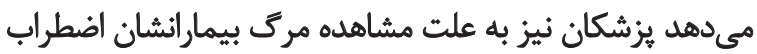

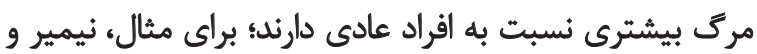
همكاران [If]

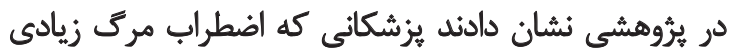

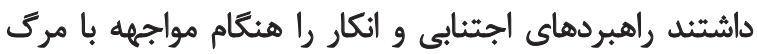

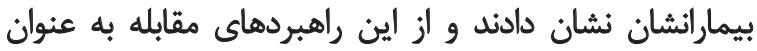

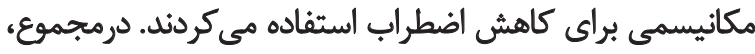

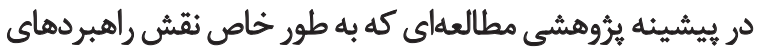

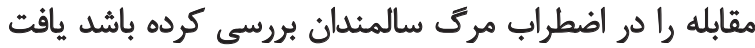

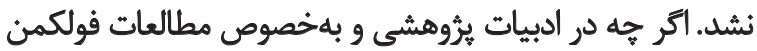
و همكاران [rع] نوعى توافق كلى مبنى بر بر تأثير منفى مقابله

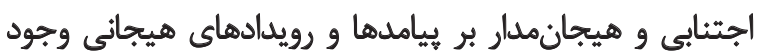

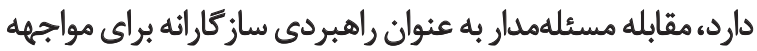

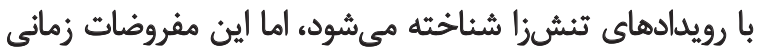

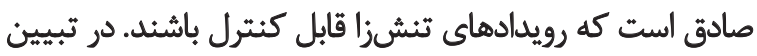

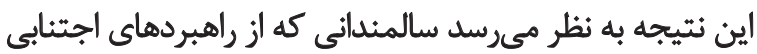

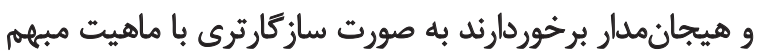

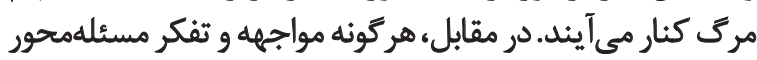

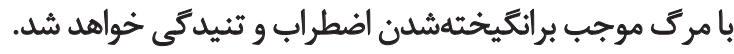

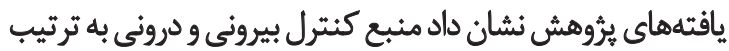

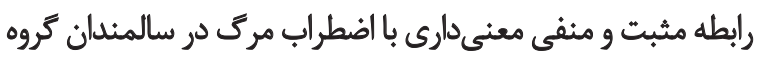

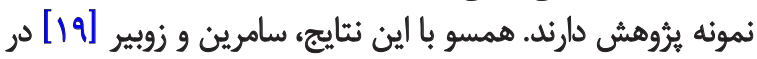

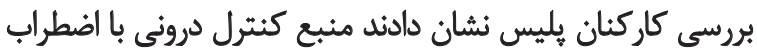

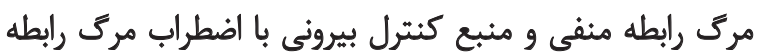

هحاسبهشده در سطح اطمينان 99 درصد معني دار است. با توجه

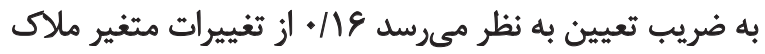

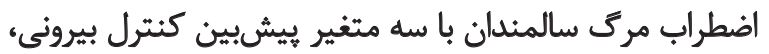

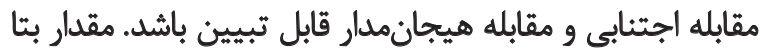
نشان مي دهد كنترل بيرونى به صورت مستقيمه و مقابله اجتنابي و و

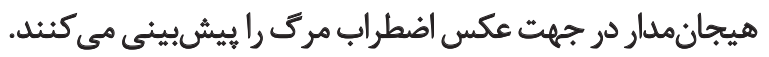

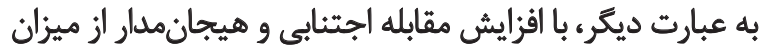

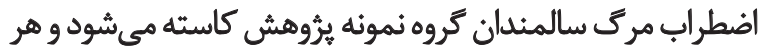

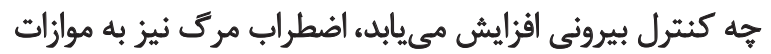

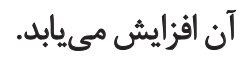

ث

اين مطالعه با هلف بيشئينى اضطراب مركى سالمندان بر مابر

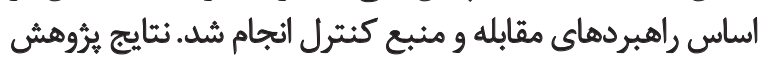

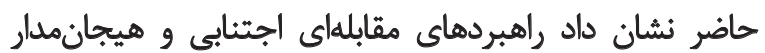

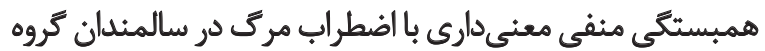

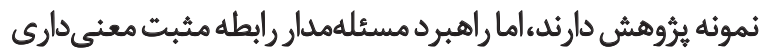

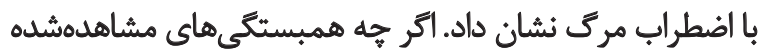

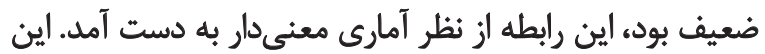

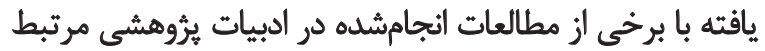

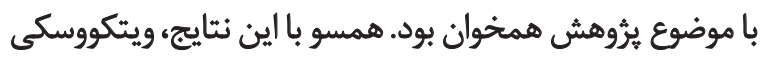

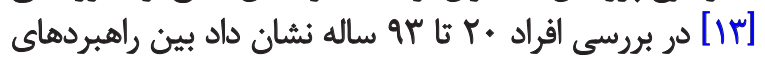

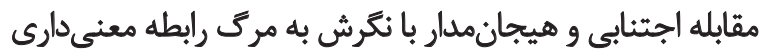

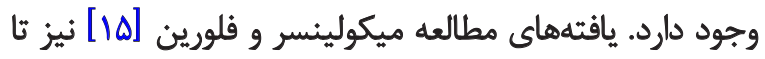

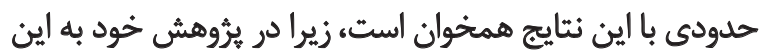

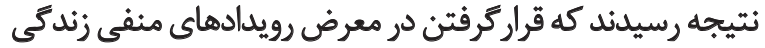


در سطح نظرى، يافتههاى مطالعه حاضر ثأييدى بر

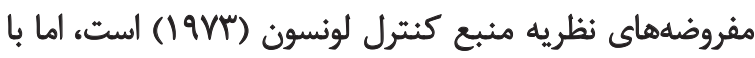

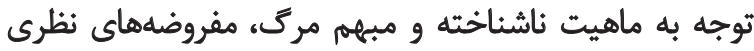

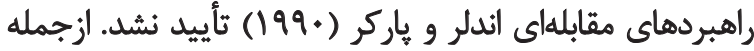

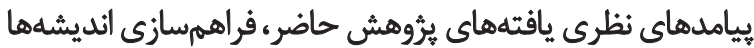

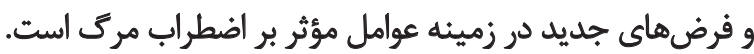

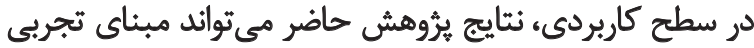

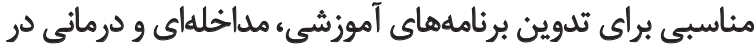
زمينه اضطراب مرك سالمندان باشد.

از محدويتهاى بثوهش حاضر مى توان به هايينبودن سطح

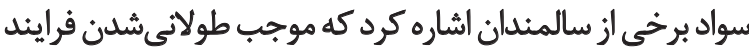

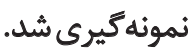

با توجه به يافتهاي يُورهش بيشنهاد مي شود به منظور افرزايش

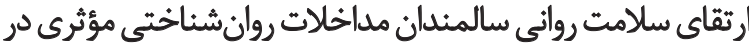

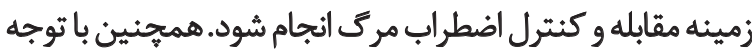

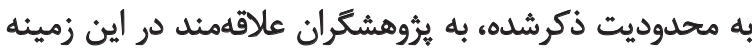

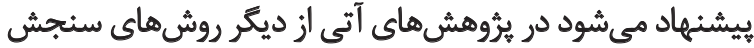

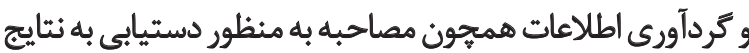

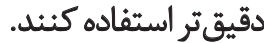

$$
\text { تشكر و قدردانى }
$$

از كليه سالمندان محترم شركت كننده در يثوهش حاضر كه

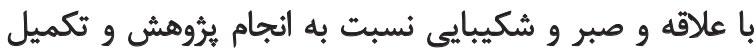

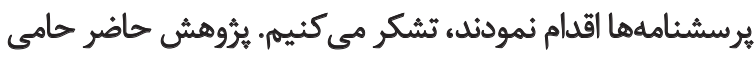

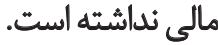

مثبت معنى دارى دارد. نتايج تحليل ركرسيون نشان داد منبع كنترل

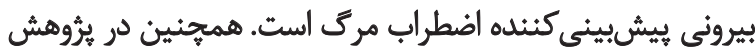

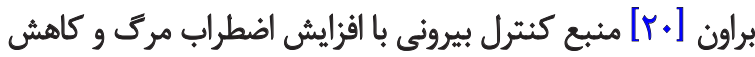

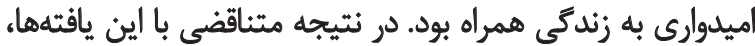

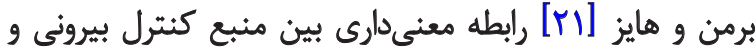

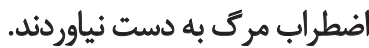

دليل تناقض يافتهها را ميتوان به روششناسى متفاوت

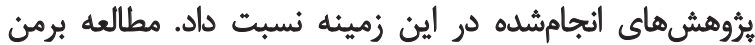

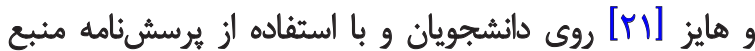

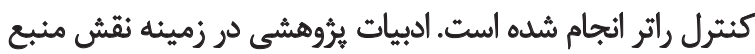

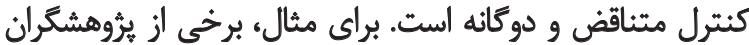

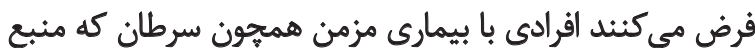

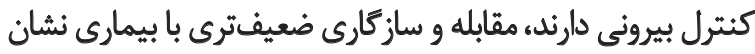

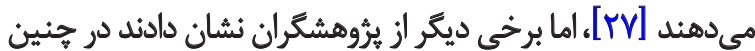

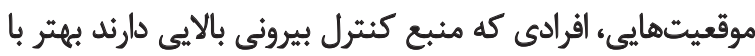

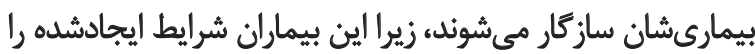

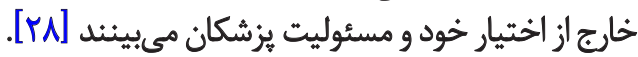
لونسون (IVIT) بر اين باور است افرادى كه منبع كنترل

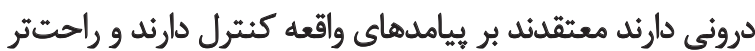

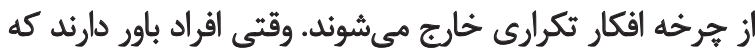

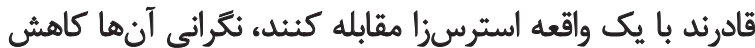

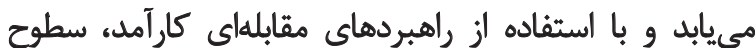

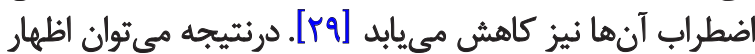

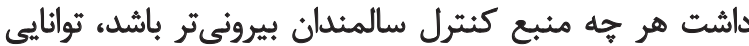

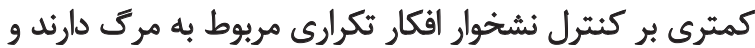

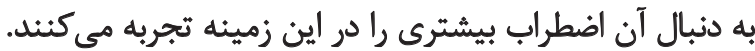

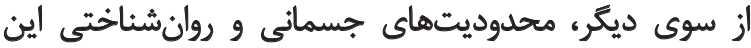

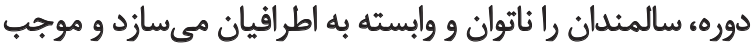

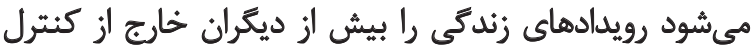

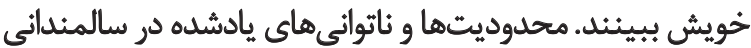

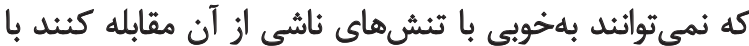

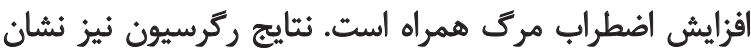

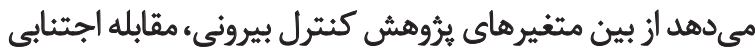

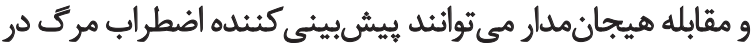

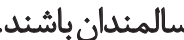

\section{نتيجلة كيرى نهايى}

در دوره سالمندى افراد بائزديكشدن به بايان حيات، مشكلاتى إنى

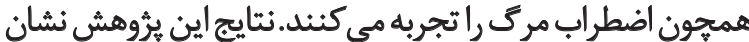

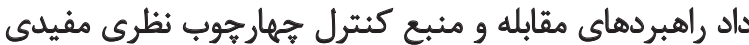

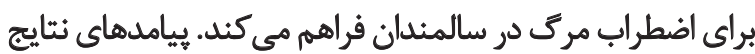
يرؤهش حاضر را مى توان در دو سطح نظرى و كاربردى به شرح

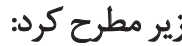




\section{References}

[1] Mejia M, Hyman SM, Behbahani S, Farrell-Turner K. Death anxiety and ageist attitudes are related to trainees' interest in working with older adults. Gerontology \& Geriatrics Education. 2016; 1-16. doi: 10.1080/02701960.2016.1247063

[2] Potes A, Gagnon G, Touré EH, Perreault M. Patient and clinician assessments of symptomatology changes on older adults following a psycho-educational program for depression and anxiety. Psychiatric Quarterly. 2016; 87(4):649-62. doi: 10.1007/s11126016-9416-4

[3] Hui VKY, Coleman PG. Afterlife beliefs and ego integrity as two mediators of the relationship between intrinsic religiosity and personal death anxiety among older adult british christians. Research on Aging. 2012; 35(2):144-62. doi: 10.1177/0164027512436429

[4] McClatchey IS, King S. The impact of death education on fear of death and death anxiety among human services students. Journal of Death and Dying. 2015; 71(4):343-61. doi: $10.1177 / 0030222815572606$

[5] Momtaz YA, Haron SA, Ibrahim R, Hamid TA. Spousal death anxiety in old age. Journal of Death and Dying. 2015; 72(1):69-80. doi: $10.1177 / 0030222815574702$

[6] Chaiwutikornwanich A. Belief in the after life, death anxiety, and life satisfaction of buddhists and christians in Thailand: Comparisons between different religiosity. Social Indicators Research. 2014; 124(3):1015-32. doi: 10.1007/s11205-014-0822-4

[7] Garbay M, Gay MC, Claxton-Oldfield S. Motivations, death anxiety, and empathy in hospice volunteers in France. American Journal of Hospice and Palliative Medicine. 2014; 32(5):521-7. doi: 10.1177/1049909114536978

[8] Aboh JU, Nwankwo BE, Agu SA, Chikwendu CE, Obi TC. Locus of control, death anxiety and length of service of electricity workers in Nigeria. IFE Psychologia: An International Journal. 2015; 23(1):197-203

[9] McKenzie EL, Brown PM, Mak AS, Chamberlain P. 'Old and ill': Death anxiety and coping strategies influencing health professionals' well-being and dementia care. Aging \& mental health. 2017; 21(6):634-41. doi: 10.1080/13607863.2016.1144711

[10] Kobau R, Seligman MEP, Peterson C, Diener E, Zack MM Chapman D, et al. Mental health promotion in public health: Perspectives and strategies from positive psychology. American Journal of Public Health. 2011; 101(8):1-9. doi: 10.2105/ ajph.2010.300083

[11] Kronenberg LM, Goossens PJJ, van Busschbach J, van Achterberg T, van den Brink W. Coping styles in substance use disorder (SUD) patients with and without co-occurring Attention Deficit/ Hyperactivity Disorder (ADHD) or autism spectrum disorder (ASD). BMC Psychiatry. 2015; 15(1). doi: 10.1186/s12888-0150530-x

[12] Marquez-Arrico JE, Benaiges I, Adan A. Strategies to cope with treatment in substance use disorder male patients with and without schizophrenia. Psychiatry Research. 2015; 228(3):752-9. doi: 10.1016/j.psychres.2015.05.028

[13] Wittkowski J. Coping and attitudes toward dying and death in German adults. Journal of Death and Dying. 2015; 72(4):316-39. doi: $10.1177 / 0030222815575283$
[14] Neimeyer GJ, Behnke M, Reiss J. Constructs and coping: Physicians' responses to patient death. Death Education. 1983; 7(23):245-64. doi: $10.1080 / 07481188308252165$

[15] Mikulincer M, Florian V. Stress, coping, and fear of personal death: The case of middle-aged men facing early job retirement. DeathStudies.1995;19(5):413-31.doi:10.1080/07481189508253391

[16] Julius Ogunleye A. Health locus of control, death anxiety and risky sexual behavior among undergraduate students in $\mathrm{Ni}$ geria. Psychology and Behavioral Sciences. 2015; 4(2):51-7. doi: 10.11648/j.pbs.20150402.13

[17] Ben-Ari OT, Florian V, Mikulincer M. The impact of mortality salience on reckless driving: A test of terror management mechanisms. Journal of Personality and Social Psychology. 1999; 76(1):35-45. doi: 10.1037/0022-3514.76.1.35

[18] O'Connor DB, Shimizu M. Sense of personal control, stress and coping style: A cross-cultural study. Stress and Health. 2002; 18(4):173-83. doi: 10.1002/smi.939

[19] Samreen H, Zubair A. Locus of control and death anxiety among police personnel. Pakistan Journal of Psychological Research. 2013; 28(2):261-275.

[20] Brown AJ, Sun CC, Urbauer DL, Bodurka DC, Thaker PH, Ramondetta LM. Feeling powerless: Locus of control as a potential target for supportive care interventions to increase quality of life and decrease anxiety in ovarian cancer patients. Gynecologic Oncology. 2015; 138(2):388-93. doi: 10.1016/j.ygyno.2015.05.005

[21] Berman AL, Hays JE. Relation between death anxiety, belief in afterlife, and locus of control. Journal of Consulting and Clinical Psychology. 1973; 41(2):318. doi: 10.1037/h0035122

[22] Tabachnick BG, Fidell LS, Osterlind SJ. Using multivariate statistics. Boston: Pearson Education; 1983.

[23] Piri L, Shararay M. [The relationship between identity style, assertive and coping styles with mental pressure (Persian)] Journal of Psychological Studies. 2005; 1(2):39-54. doi: 10.22051/ PSY.1970.1660

[24] Farahani MN. The relationship of locus of control, extraversion neuroticism with the academic achievement of Iranian students Wales: University of New South Wales; 1994.

[25] Rajabi G, Bohrani M. [Item factor analysis of death anxiety scale (Persian)]. Journal of Psychology. 2001; 20:331-44.

[26] Folkman S, Chesney M, McKusick L, Ironson G, Johnson DS, Coates TJ. Translating coping theory into an intervention. The Social Context of Coping. 1991; 239-60. doi: 10.1007/978-1-48993740-7_11

[27] Coughlin AM, Badura AS, Fleischer TD, Guck TP. Multidisciplinary treatment of chronic pain patients: Its efficacy in changing patient locus of control. Archives of Physical Medicine and Rehabilitation. 2000; 81(6):739-40. doi: 10.1016/s0003-9993(00)90103-5

[28] Broers S, Kaptein AA, Le Cessie S, Fibbe W, Hengeveld MW. Psychological functioning and quality of life following bone marrow transplantation. Journal of Psychosomatic Research. 2000; 48(1):11-21. doi: 10.1016/s0022-3999(99)00059-8

[29] Moulding R, Kyrios M. Anxiety disorders and control related beliefs: The exemplar of Obsessive-Compulsive Disorder (OCD) Clinical Psychology Review. 2006; 26(5):573-83. doi: 10.1016/j. cpr.2006.01.009 